\title{
Value of insoluble PABPN1 accumulation in the diagnosis of oculopharyngeal muscular dystrophy
}

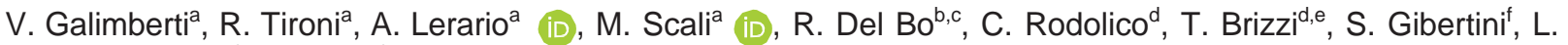 \\ Maggif, M. Mora ${ }^{f}$, A. Toscano ${ }^{d}$, G. P. Comi ${ }^{b, c}$, M. Sciacco ${ }^{a}$, M. Moggio and L. Peverellia \\ ${ }^{a}$ Neuromuscular and Rare Disease Unit, Department of Neuroscience, Foundation IRCCS Ca' Granda Ospedale Maggiore Policlinico, \\ Milano; 'Dino Ferrari Centre, Neuroscience Section, Department of Pathophysiology and Transplantation (DEPT), University of Milan, \\ Milano; 'Neurology Unit, IRCCS Foundation Ca' Granda Ospedale Maggiore Policlinico, Milano; ${ }^{\mathrm{d} N e u r o l o g y}$ and Neuromuscular \\ Diseases Unit, Department of Clinical and Experimental Medicine, University of Messina, Messina; ${ }^{\mathrm{e} D I B I M I S}$ University of Palermo, \\ Palermo; and ${ }^{\mathrm{f}}$ Neuromuscular Diseases and Neuroimmunology Unit, Fondazione IRCCS Istituto Neurologico Carlo Besta, Milano, Italy
}

\section{Keywords:}

oculopharyngeal

musclular dystrophy, PABPN1 accumulations, PABPN1

immunofluorescence, rimmed vacuoles, tubulofilamentous intranuclear inclusions
Background and purpose: The aim was to assess the value of insoluble PABPN1 muscle fibre nuclei accumulation in the diagnosis of atypical cases of oculopharyngeal muscular dystrophy (OPMD).

Methods: Muscle biopsies from a selected cohort of 423 adult patients from several Italian neuromuscular centres were analysed by immunofluorescence: 30 muscle biopsies of genetically proven OPMD, 30 biopsies from patients not affected by neuromuscular disorders, 220 from genetically undiagnosed patients presenting ptosis or swallowing disturbances, progressive lower proximal weakness and/or isolated rimmed vacuoles at muscle biopsy and 143 muscle biopsies of patients affected by other neuromuscular diseases.

Results: The detection of insoluble nuclear PABPN1 accumulation is rapid, sensitive (100\%) and specific (96\%). The revision of our cohort allowed us to discover 23 new OPMD cases out of 220 patients affected with nonspecific muscle diseases.

Conclusions: Oculopharyngeal muscular dystrophy is often misdiagnosed leading to diagnosis delay, causing waste of time and resources. A great number of these cases present symptoms and histological findings frequently overlapping with other muscle diseases, i.e. inclusion body myositis and progressive external ophthalmoplegia. PABPN1 nuclear accumulation is a reliable method for diagnostic purposes and it is safe and useful in helping pathologists and clinicians to direct genetic analysis in the case of suspected OPMD, even when clinical and histological clues are deceptive.

\section{Introduction}

Oculopharyngeal muscular dystrophy (OPMD) is a late-onset muscle disease, clinically characterized by ptosis, with or without ophthalmoparesis, dysphagia and proximal weakness. Inheritance can be autosomal dominant or recessive [1-3]. OPMD is caused by a GCN repeat expansion in the poly-A binding protein nuclear 1 gene (PABPN1) (14q11.2-q13), which leads

Correspondence: Lorenzo Peverelli, Fondazione IRCCS Ca’ Granda Ospedale Maggiore Policlinico, Padiglione Ex convitto suore piano 1, Via Francesco Sforza 35 20122, Milano, Italia (tel.: +39

0255036504; fax: +39 0255033827; e-mail:

lorenzo.peverelli84@gmail.com). to an expanded polyalanine tract in the N-terminal of the PABPN1 protein. Normal repeat size is (GCN)10, and (GCN)11-18 is considered pathological. Poly-A expansion in PABPN1 favours the accumulation of toxic, insoluble protein deposits in the nuclei of muscle cells [4]. The point mutation c.35G $>C$ in $P A B P N 1$ can also affect disease phenotype [1].

Oculopharyngeal muscular dystrophy is often overlooked and diagnosed after considerable delay, prolonging discomfort for patients and their relatives and wasting time and resources [6,7]. Histopathological findings of muscle biopsies from affected patients are overall nonspecific; however, rimmed vacuoles and oxidative stain alterations along with scattered 
mitochondrial abnormalities can be detected [5]. Symptoms and histological findings of OPMD frequently overlap with those of other muscle diseases, including inclusion body myositis (IBM) and progressive external ophthalmoplegia [5-8]. In these unclear cases, identification of insoluble PABPN1 nuclear aggregates in skeletal muscle biopsy can help confirm OPMD diagnosis. The detection of PABPN1 inclusions by immunofluorescence is a standardized technique but so far is used mainly for research purposes [9-11].

To verify whether nuclear accumulation of insoluble PABPN1 is a reliable diagnostic marker in suspected OPMD, muscle biopsy samples were analysed from 423 patients whose tissues were stored in several biobanks.

\section{Materials and methods}

All patients were recruited between April 2010 and February 2017 after written informed consent was obtained, in compliance with Italian law. The study protocol and consent forms were approved by the ethics committee and were in agreement with biobank regulations (Telethon Network for Genetics Biobanks and Eurobiobank). This study was performed in accordance with ethical standards as laid down in the 1964 Declaration of Helsinki.

\section{Patients}

Thirty muscle biopsies from patients with genetically determined OPMD and 30 muscle specimens without detectable histopathological alterations were analysed. Also, 220 genetically undiagnosed patients presenting one or more of the following features were selected: ptosis or dysphagia, progressive lower proximal weakness and/or isolated rimmed vacuoles at muscle biopsy. Amongst these, 131 had isolated ptosis or dysphagia without rimmed vacuoles (group I), 41 had isolated progressive lower proximal weakness or dysphagia and rimmed vacuoles (group II) and 48 patients were selected because of the presence of isolated rimmed vacuoles (group III). In addition, 143 muscle biopsies from patients affected with other, previously diagnosed, neuromuscular diseases were analysed (Table 1).

Patients with polymyositis and dermatomyositis met the clinical criteria of Dalakas and Hohlfeld [12], and patients with immune-mediated necrotizing myopathies met the criteria defined at the 224th European Neuromuscular Centre (ENMC) International Workshop in 2016 [13]. IBM was defined using recently reported criteria [14], and in all other cases genetic analyses were performed to confirm the diagnosis.
Table 1 Details of 143 muscle biopsies from patients affected with other neuromuscular diseases

\begin{tabular}{lr}
\hline Neuromuscular disease & Number of patients \\
\hline Myotonic dystrophy type 1 (DM1) & 10 \\
Proximal myotonic myopathy & 5 \\
Facio-scapulo-humeral dystrophy & 10 \\
Limb-girdle muscular dystrophy & 8 \\
Duchenne muscular dystrophy & 6 \\
Becker muscular dystrophy & 7 \\
Polymyositis & 13 \\
Dermatomyositis & 6 \\
Inclusion body myositis & 10 \\
Immune-mediated necrotizing myopathies & 7 \\
Mitochondrial myopathy & 11 \\
Glycogenosis & 6 \\
Lipid metabolism disorder & 8 \\
Myofibrillar myopathy & 10 \\
Congenital myopathy & 5 \\
Congenital dystrophy & 5 \\
Valosin-containing protein myopathy & 1 \\
Neuropathies & 143 \\
Total & \\
\hline
\end{tabular}

\section{Muscle biopsy}

Muscle specimens came from the Biobank of Skeletal Muscle, Peripheral Nerve, DNA and Cell Cultures (IRCCS Ca' Granda Ospedale Maggiore Policlinico, Milano), the Biobank of Cells, Tissues and DNA from Patients with Neuromuscular Diseases (Fondazione IRCCS Istituto Neurologico C. Besta, Milano) and the Department of Clinical and Experimental Medicine, University of Messina. Most muscle specimens were obtained from the biceps brachii (375 out 423), and the rest of them (48) from the quadriceps.

\section{Molecular genetic testing and analysis}

All the 423 patients of the study were screened for OPMD mutations (both GCN repeats and point mutation c.35G>C).

Genomic DNA was extracted from peripheral blood or muscle biopsy specimens using standard procedures. The PABPN1 gene polyalanine codon expansion was amplified by polymerase chain reaction (PCR) using FastStart TAQ DNA Polymerase (Roche, Basel, Switzerland) and sized by capillary electrophoresis on an ABI PRISM $3130 \mathrm{XL}$ genetic analyser (Thermo Fisher Scientific, Waltham, MA, USA). The size standard was the GeneScan 500 LIZ (Applied Biosystems, Thermo Fisher Scientific, Waltham, MA, USA), and GeneMapper v.5.0 software (Thermo Fisher Scientific, Waltham, MA, USA) was used for analysis. 
Unlabelled primers were used for PCR amplification of exon 1 of $P A B P N 1$. Sequence analysis was performed in both forward and reverse directions using ABI Big Dye Terminator Cycle Sequencing (version 3.1, Thermo Fisher Scientific, Waltham, MA, USA) in an ABI PRISM 3130 XL genetic analyser [15].

\section{Light microscopy and immunofluorescence}

Cryostatic sections from frozen skeletal muscle specimens were stained with modified G€ m€ ri trichrome for histological evaluation and examined under immunofluorescence (see Appendix S1 and Table S1 for details).

For fluorescence microscopy, a Leica DM 4000B was used with an excitation filter BP 300-385 nm, DM $400 \mathrm{~nm}$ and BA $420 \mathrm{~nm}$ for $4^{0}$,6-diamidino-2-phenylindole (DAPI) and an excitation filter BP 450-480 nm. Images at 409 original magnification were acquired with a Leica DFC 420C digital camera using Leica Application Suite software (version 4.6.2). The percentage of muscle fibre nuclei positive for PABPN1 aggregates was calculated by comparing the number of positive nuclei to the total number of myonuclei on each section. Nuclear PABPN1 aggregates were counted in immunolabelled cross-sections, using ImageJ (Scion Corporation, Bethesda, MD, USA). Samples showing evidence of insoluble nuclear fluorescence foci of PABPN1 associated with a positive genetic test were considered to be positive for OPMD.

\section{Results}

This study included samples from 30 genetically determined OPMD patients and 30 healthy patients. As shown in Fig. 1, all samples from genetically diagnosed OPMD patients contained intense fluorescent foci in muscle tissue nuclei (30/30). Conversely, protein nuclear immunoreactivity was absent from muscle
Table 2 Oculopharyngeal muscular dystrophy genetic assessment results of the newly diagnosed patients

\begin{tabular}{lc}
\hline GCN expansion & Number of patients \\
\hline $10 / 11$ GCN & 1 \\
$10 / 12$ GCN & 3 \\
$10 / 13$ GCN & 10 \\
$10 / 14$ GCN & 3 \\
$11 / 11$ GCN & 4 \\
$11 / 12$ GCN & 1 \\
c35G>C & 1 \\
Total & 23
\end{tabular}

tissues of patients without any neuromuscular abnormalities (OPMD genetic analysis was also negative) (30/30).

A total of 220 undiagnosed cases were classified into three groups, as described in Materials and methods. Thirteen patients from group I and 10 from group II were positive on both immunofluorescence and genetic analysis for OPMD. In addition, four patients from group I, two from group II and four from group III showed only nuclear accumulation (false-positive cases), whereas 183 patients had negative findings with both analyses. Table 2 summarizes the GCN repeats and mutations in PABPN1 found in the 23 newly diagnosed cases from the 220 patients.

The nuclear distribution of PABPN1 was also evaluated by immunofluorescence analysis in 143 muscle biopsies from patients with other genetically proven neuromuscular diseases. Intranuclear PABPN1-positive foci were detected in six muscle specimens out of 143 (4\%) from patients with myotonic dystrophy type 1 (DM1) (two cases), two from polymyositis specimens, one from a patient with carnitine palmitoyl transferase II deficiency and one from a case of IBM associated with Paget disease of bone and frontotemporal dementia (IBMPFD). Table 3 summarizes the results for the entire cohort.
Figure 1 PABPN1 aggregation in the nuclei of muscle fibres from one OPMD case and one control. Muscle tissues from an OPMD sample and from a negative control case were stained with a monoclonal anti-PABPN1 antibody (EPY3000-Abcam ab75855), followed by a goat anti-rabbit cy3 secondary antibody. Blue: DAPI staining. Bar: $50 \mu \mathrm{m}$. Arrows indicate intranuclear PABPN1 accumulation brightness in comparison to all other nuclei. Also, a few nuclei show stronger nuclear stain in the OPMD sample than in the control

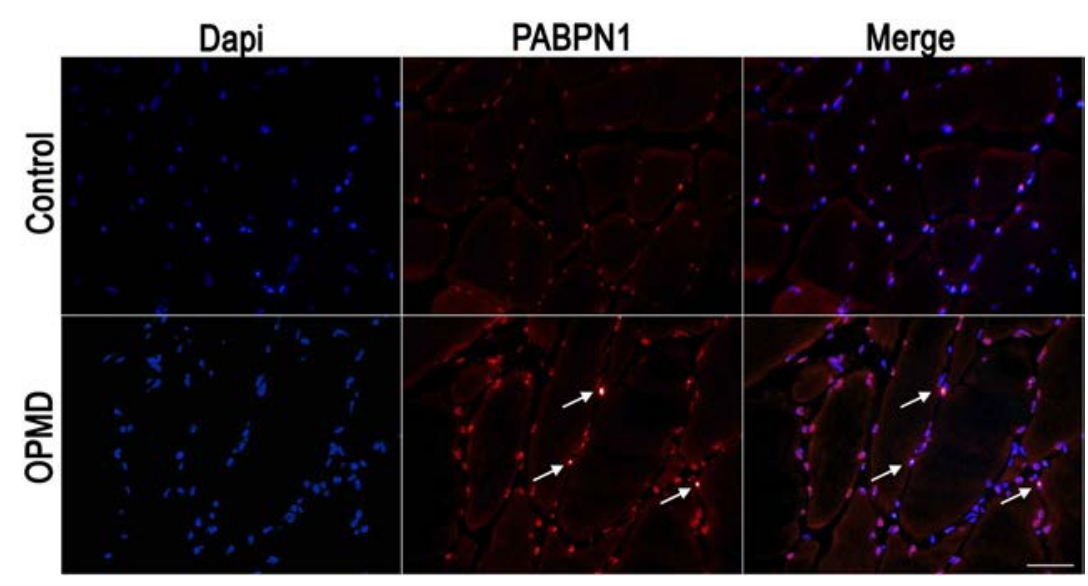


Table 3 Histological and genetics findings amongst the tested patients

\begin{tabular}{|c|c|c|c|}
\hline & $\begin{array}{l}\text { Number } \\
\text { of total } \\
\text { biopsies }\end{array}$ & $\begin{array}{l}\text { Number of } \\
\text { biopsies positive } \\
\text { for } \\
\text { immunofluorescent } \\
\text { PABPN1 foci }\end{array}$ & $\begin{array}{l}\text { Number of } \\
\text { biopsies } \\
\text { positive on } \\
\text { genetic } \\
\text { analysis for } \\
\text { GCN } \\
\text { expansion }\end{array}$ \\
\hline $\begin{array}{l}\text { Confirmed OPMD } \\
\text { Normal }\end{array}$ & $\begin{array}{l}30 \\
30\end{array}$ & $\begin{array}{r}30 \\
0\end{array}$ & $\begin{array}{r}30 \\
0\end{array}$ \\
\hline $\begin{array}{l}\text { Undiagnosed } \\
\text { neuromuscular } \\
\text { diseases }\end{array}$ & 220 & & \\
\hline $\begin{array}{l}\text { Group I (clinical } \\
\text { signs) }\end{array}$ & 131 & 17 & 13 \\
\hline $\begin{array}{l}\text { Group II (clinical } \\
\text { and } \\
\text { histopathology } \\
\text { signs) }\end{array}$ & 41 & 12 & 10 \\
\hline $\begin{array}{l}\text { Group III } \\
\text { (histopathology } \\
\text { signs) }\end{array}$ & 48 & 4 & 0 \\
\hline $\begin{array}{l}\text { Other } \\
\text { neuromuscular } \\
\text { diseases }\end{array}$ & 143 & 6 & 0 \\
\hline
\end{tabular}

In all genetically confirmed cases (30 already known, 23 newly discovered), the proportion of positive nuclei was $>3 \%$ (median percentage $4.4 \%$ ). No correlation was found between percentage of immunopositive nuclei and repeat number, clinical severity and time from onset of the disease.

In 14 out of 16 false-positive cases, the percentage of positive nuclei was $<1.5 \%$ (median percentage $0.9 \%$ ). Only in the two polymyositis cases the percentage of positive nuclei was $>3 \%$.

\section{Discussion}

The clinical features of OPMD show some overlap with other neuromuscular diseases such as KearnsSayre syndrome [8], myasthenia gravis, oculopharyngodistal myopathy [7], blepharophimosis with ptosis and epicanthus inversus syndrome [16] and IBM [5], which makes pinpointing the correct diagnosis a challenge. The OPMD diagnosis is based on genetic analysis with sequencing of the first exon of PABPN1 to determine the size of GCN trinucleotide repeats or the presence of the c.35G $>$ C mutation. In the era of nextgeneration sequencing, OPMD represents an exception because of the difficulties in detecting small DNA insertions (such as 11-18 GCN repetitions), possibly missing the diagnosis [17]. Therefore, although the Sanger method is pivotal in diagnosing OPMD, muscle biopsy along with electromyography plays a crucial role in differential diagnosis especially in the case of atypical and/or mild clinical presentations, possibly associated with an unclear family history.

Muscle biopsy in OPMD does not show typical dystrophic features. Instead, several angulated type 1 fibres and rimmed vacuoles are detected in a range from $0.1 \%$ to $3.5 \%$ of the fibres [18]. Electron microscopy shows intranuclear tubular filaments in no more than $3 \%-6 \%$ of the nuclei [19]. Other nonspecific occasional morphological findings include 'motheaten' or whorled fibres, core-like areas that are best detected with nicotinamide adenine dinucleotide dehydrogenase (NADH) staining, and cytochrome c oxidase (COX) deficient fibres, sometimes with an associated increase in succinate dehydrogenase activity (e.g. ragged red fibres) (Fig. 2) [5]. These scattered histological clues are sometimes hard to detect and elusive even to trained pathologists. In our cohort, the most frequent alterations detected were COX-negative fibres, rimmed vacuoles and NADH-staining alterations (see Appendix S1 and Table S1).

To date, the detection of nuclear accumulation of insoluble PABPN1 by immunofluorescence has been performed primarily for research purposes and rarely for diagnosis [20]. In a recent, prospective observational study involving a small population of patients with genetically confirmed OPMD, nuclear insoluble PABPN1 accumulations were detected in muscle fibres before clinical onset [21]. Given these data, a large cohort of undiagnosed patients was selected presenting one or more features, including ptosis or dysphagia, isolated lower proximal muscle weakness and/or rimmed vacuoles at muscle biopsy, and intranuclear insoluble PAPBPN1 accumulations were searched for in skeletal muscle specimens.

The high frequency (10.4\%) of new OPMD cases diagnosed in patients with early clinical symptoms and no positive familiar history emphasizes the relevance of this diagnostic tool that relies on a quick and simple immunofluorescence test. This technique helps reduce diagnostic delay, allows for early diagnosis in oligosymptomatic individuals and prevents diagnostic errors, especially in cases involving misleading clinical symptoms and mitochondrial alterations at muscle biopsy. Faster diagnosis allows patients and their relatives to seek early genetic counselling, which can be crucial for quality of life and family planning. Finally, it is also resource saving.

The 41 biopsies of group II were characterized by nonspecific clinical and histological signs of neuromuscular disease. The immunofluorescence study with anti-PABPN1 antibodies allowed a diagnosis of OPMD to be made in 10 of 41 of these patients 
Figure 2 Morphological findings in muscle biopsies from three OPMD cases. Genotype (GCN)10/(GCN)13 in all of them. (a), (b) Case 1: (a) Modified G€ m€ ri trichrome (MGT) stain shows an almost normal pattern whereas (b) cytochrome c oxidase/succinate dehydrogenase (COX/SDH) combination staining shows scattered COX-negative fibres sometimes with increased SDH activity (ragged red fibres). (c) Case 2: MGT shows a more typical OPMD pattern characterized by fibre calibre variability, rimmed vacuoles and increased endomysial connective tissue. (d) Case 3: Nicotinamide adenine dinucleotide (NADH) staining reveals 'core-like' areas and dark stain in hypotrophic fibres. (a), (c), (d) $100 \mu \mathrm{m}$ bar. (b) $200 \mu \mathrm{m}$ bar
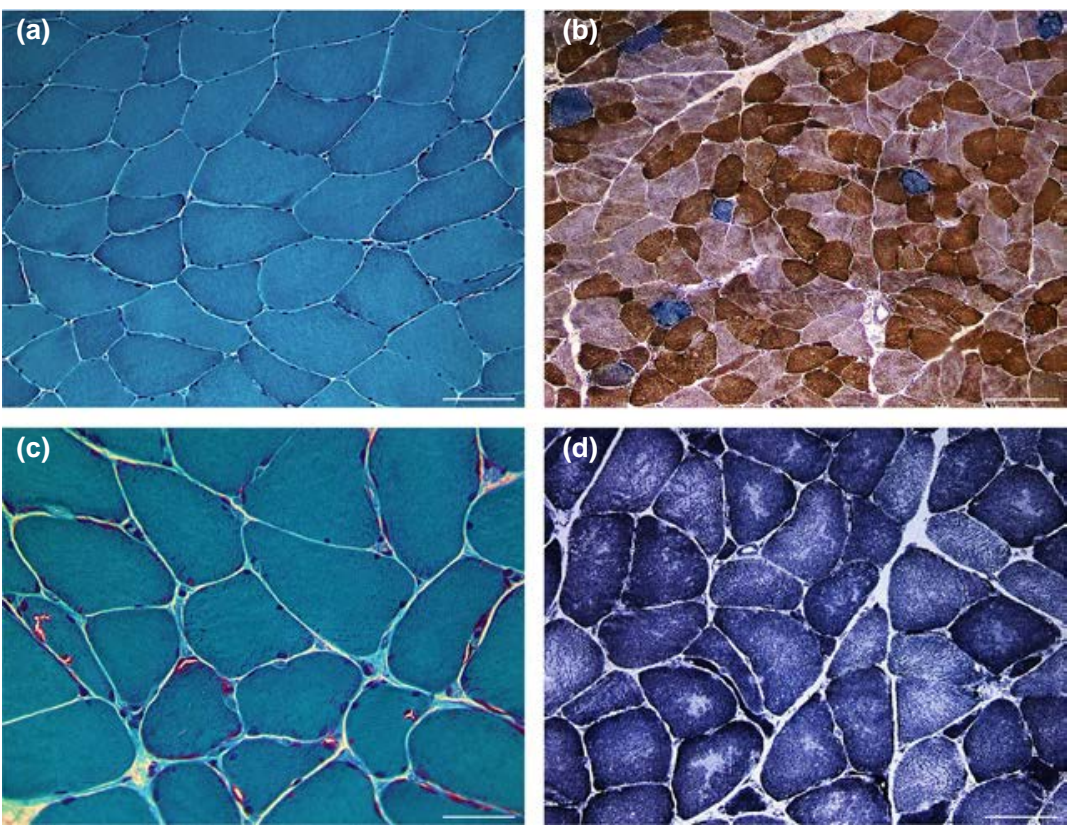

(24.3\%). Despite the presence of rimmed vacuoles that could have been a hint for OPMD, in several of these cases other diagnostic pathways fulfilling clinical and histological data were previously studied. In group III, none of the patients with isolated rimmed vacuoles at muscle biopsy and no suggestive clinical symptoms had confirmed OPMD. These data show that rimmed vacuoles alone are not necessarily pathognomonic; indeed, they more often appear as nonspecific findings in advanced OPMD disease; moreover, rimmed vacuoles are present in other myopathies, especially IBM [5].

Finally, 10 out of 220 (4.5\%) samples from patients with undiagnosed neuromuscular diseases (belonging to the previous groups) were positive for PABPN1 nuclear inclusions despite being genetically negative for OPMD (false-positive cases). It is not excluded that mutations or alterations in some as-yet unknown upstream or downstream regulatory factors influence PABPN1 in these patients.

The percentage of positive muscle nuclei was also evaluated in all muscle samples, finding that positivity $>3 \%$ was strongly suggestive of OPMD. A lower percentage of immunoreactive nuclei (particularly under $1.5 \%$ ) was highly indicative of false-positive cases.

Insoluble PABPN1 nuclear accumulation has been reported in other non-OPMD disorders such as IBMPFD [20]. Therefore, our study was expanded to 143 biopsies from patients affected with other neuromuscular diseases. A distinctive intranuclear staining of PABPN1 was found in six cases (4.1\%), specifically in two DM1, two polymyositis, one IBMPFD and one lipid storage myopathy due to carnitine palmitoyl transferase II deficiency. Genetic analysis for DM1 $(D M P K)$, IBMPFD (VCP) and lipid storage myopathy due to carnitine palmitoyl transferase II deficiency was performed on the 10 false-positive cases but yielded no positives for mutations in the above-mentioned genes. Also no evidence of inflammation was found, ruling out the possibility of undiagnosed polymyositis.

Myotonic dystrophy type 1 is caused by the accumulation of expanded, non-coding RNAs. Like OPMD, it is considered a neurodegenerative RNA pathology [22]. Positivity for nuclear insoluble aggregation of PABPN1 in DM1 muscle samples could be the result of a nonspecific linkage between abnormal nuclear mRNA accumulations and PABPN1 residues [23-25]. However, positivity was found in only two of 10 DM1 samples and none was detected in other spliceopathies examined in this cohort (i.e. TDP43 mutations). In addition, no such overlap has been reported in the literature. Matsubara et al. have proposed that the presence of PABPN1-positive staining in IBMPFD is the result of intranuclear inclusions with palisading tubulofilaments [20], as commonly observed in this disease. To the best of our knowledge, no other hypothesis can be found in the literature that explains PABPN1 accumulation in other neuromuscular diseases.

There is no evidence about any specific immunostaining marker able to differentiate intranuclear PABPN1 accumulation in OPMD from those detected 
in other diseases (DM1, IBMPFD, polymyositis and lipid storage myopathy due to carnitine palmitoyl transferase II deficiency).

This review of samples allowed 23 new OPMD cases to be uncovered in previously undiagnosed or misdiagnosed patients. The most frequent alternative diagnosis was of mitochondrial disease, because of the presence of supportive clinical findings and oxidative stain abnormalities (Fig. 2a and b). Based on these results, it is suggested that PABPN1 accumulations are looked for in muscle biopsies in all clinical cases involving ptosis with or without ophthalmoparesis or dysphagia, progressive lower proximal weakness and mild mitochondrial signs.

In conclusion, PABPN1 accumulation detection is rapid, specific (96\%), sensitive (100\%) and extremely useful in properly addressing both molecular and biochemical confirmation analyses. It can therefore be regarded as a reliable, preliminary OPMD diagnostic marker when clinical and histological clues are unclear.

\section{Acknowledgements}

The authors thank the Associazione Amici del 'Centro Dino Ferrari', University of Milan, the Biobank of Skeletal Muscle, Peripheral Nerve, DNA and Cell Cultures, and the Telethon Network of Genetic Biobanks. Dr Capucine Trollet and Francesco Nozzolino are also thanked for professional and technical support.

\section{Disclosure of conflicts of interest}

The authors have no financial interest to report and did not receive any funding.

\section{Supporting Information}

Additional Supporting Information may be found in the online version of this article:

Appendix S1. Detailed material and methods; immunofluorescence on muscle slides.

Table S1. Clinical and histopathological details of genetically confirmed OPMD patients

\section{References}

1. Garibaldi M, Pennisi EM, Bruttini M, et al. Droppedhead in recessive oculopharyngeal muscular dystrophy. Neuromuscul Disord 2015; 25: 869-872.
2. Semmler A, Kress W, Vielhaber S, et al. Variability of the recessive oculopharyngeal muscular dystrophy phenotype. Muscle Nerve 2007; 35: 681-684.

3. Vissing J. Oculopharingeal muscular dystrophy, 2016 https://www.orpha.net/consor/cgi-bin/OC_Exp.php? Lng $=$ EN\&Expert $=270$ (15 December 2018).

4. Robinson DO, Wills AJ, Hammans SR, et al. Oculopharyngeal muscular dystrophy: a point mutation which mimics the effect of the PABPN1 gene triplet repeat expansion mutation. J Med Genet 2006; 43: e23.

5. Dubowitz V, Oldfords A, Sewry CA. Muscle Biopsy: a Practical Approach, 4th edn. London: W. B. Saunders, 2013.

6. Agarwal PK, Mansfield DC, Mechan D, et al. Delayed diagnosis of oculopharyngeal muscular dystrophy in Scotland. Br J Ophthalmol 2012; 96: 281-283.

7. Durmus H, Laval SH, Deymeer F, et al. Oculopharyngodistal myopathy is a distinct entity: clinical and genetic features of 47 patients. Neurology 2011; 76: 227235.

8. Moggio M, Colombo I, Peverelli L, et al. Mitochondrial disease heterogeneity: a prognostic challenge. Acta Myol 2014; 33: 86-93.

9. Gnocchi VF, Scharner J, Huang Z, et al. Uncoordinated transcription and compromised muscle function in the Lmna-null mouse model of Emery-Dreifuss muscular dystrophy. PLoS One 2011; 6: e16651.

10. Hett A, West S. Inhibition of U4 snRNA in human cells causes the stable retention of polyadenylated pre-mRNA in the nucleus. PLoS One 2014; 9: e96174.

11. Malerba A, Klein P, Bachtarzi $\mathrm{H}$, et al. PABPN1 gene therapy for oculopharyngeal muscular dystrophy. Nat Commun 2017; 31: 14848.

12. Dalakas MC, Hohlfeld R. Polymyositis and dermatomyositis. Lancet 2003; 362: 971-982.

13. Allenbach Y, Mammen AL, Stenzel W, et al. 224th ENMC International Workshop: Clinico-sero-pathological classification of immune-mediated necrotizing myopathies, Zandvoort, The Netherlands, 14-16 October 2016. Neuromuscul Disord 2018; 28: 87-99.

14. Rose MR. 188th ENMC International Workshop: Inclusion Body Myositis, 2-4 December 2011, Naarden, The Netherlands. Neuromuscul Disord 2013; 23: 1044-1055.

15. Brais B, Bouchard JP, Xie YG, et al. Short GCG expansions in the PABP2 gene cause oculopharyngeal muscular dystrophy. Nat Genet 1998; 18: 164-167.

16. Allen CE, Rubin PAD. Blepharophimosis-ptosis-epicanthus inversus syndrome (BPES): clinical manifestation and treatment. Int Ophthalmol Clin 2008; 48: 15-23.

17. Goodwin S, McPherson JD, Mc Combie WR. Coming of age: ten years of next-generation sequencing technologies. Nat Rev Genet 2016; 17: 333-351.

18. Engel AG. Myology, 3rd edn. New York: McGraw-Hill, 2004.

19. Tome FMS, Fardeau M. Nuclear inclusion in oculopharyngeal dystrophy. Acta Neuropathol 1980; 49: 85-87.

20. Matsubara S, Shimizu $T$, Komori $T$, et al. Nuclear inclusions mimicking poly(A)-binding protein nuclear 1 inclusions in a case of inclusion body myopathy associated with Paget disease of bone and frontotemporal dementia with a novel mutation in the valosin-containing protein gene. Neuromuscul Disord 2016; 26: 436-440.

21. Van Der Sluijs BM, Raz V, Lammens M, et al. Intranuclear aggregates precede clinical onset in 
oculopharyngeal muscular dystrophy. J Neuromuscul Dis 2016; 3: 101-109.

22. Belzil VV, Gendron TF, Petrucelli L. RNA-mediated toxicity in neurodegenerative disease. Hum Mol Genet 2013; 22: 2811-2819.

23. Bengoechea R, Tapia O, Casafont I, et al. Nuclear speckles are involved in nuclear aggregation of PABPN1 and in the pathophysiology of oculopharyngeal muscular dystrophy. Neurobiol Dis 2012; 46: 118-129.
24. Gudde AEEG, van Kessel IDG, Andre LM, et al. Trinucleotide-repeat expanded and normal DMPK transcripts contain unusually long poly(A) tails despite differential nuclear residence. Biochim Biophys Acta Gene Regul Mech 2017; 1860: 740-749.

25. Smith KP, Byron M, Johnson C, et al. Defining early steps in mRNA transport: mutant mRNA in myotonic dystrophy type I is blocked at entry into SC-35 domains. J Cell Biol 2007; 178: 951-964. 
